\title{
Maxwell consideration of polaritonic quasi-particle Hamiltonians in multi-level systems
}

Steffen Richter, Tom Michalsky, Lennart Fricke, Chris Sturm, Helena Franke, Marius Grundmann, and Rüdiger Schmidt-Grund

Citation: Appl. Phys. Lett. 107, 231104 (2015); doi: 10.1063/1.4937462

View online: https://doi.org/10.1063/1.4937462

View Table of Contents: http://aip.scitation.org/toc/apl/107/23

Published by the American Institute of Physics

\section{Articles you may be interested in}

Relaxation and emission of Bragg-mode and cavity-mode polaritons in a $\mathrm{ZnO}$ microcavity at room temperature Applied Physics Letters 95, 121102 (2009); 10.1063/1.3232228

Bosonic lasers: The state of the art (Review Article)

Low Temperature Physics 42, 323 (2016); 10.1063/1.4948614

Strong coupling between Tamm plasmon polariton and two dimensional semiconductor excitons Applied Physics Letters 110, 051101 (2017); 10.1063/1.4974901

What is the best planar cavity for maximizing coherent exciton-photon coupling Applied Physics Letters 111, 061102 (2017); 10.1063/1.4997171

Ultrastrong light-matter coupling in electrically doped microcavity organic light emitting diodes Applied Physics Letters 104, 233303 (2014); 10.1063/1.4882422

Polariton condensation in a strain-compensated planar microcavity with InGaAs quantum wells Applied Physics Letters 105, 191118 (2014); 10.1063/1.4901814

\section{Conference Proceedings}




\title{
Maxwell consideration of polaritonic quasi-particle Hamiltonians in multi-level systems
}

\author{
Steffen Richter, Tom Michalsky, Lennart Fricke, Chris Sturm, Helena Franke, \\ Marius Grundmann, and Rüdiger Schmidt-Grund \\ Institut für Experimentelle Physik II, Universität Leipzig, Linnéstr. 5, 04103 Leipzig, Germany
}

(Received 15 October 2015; accepted 27 November 2015; published online 9 December 2015)

\begin{abstract}
We address the problem of the correct description of light-matter coupling for excitons and cavity photons in the case of systems with multiple photon modes or excitons, respectively. In the literature, two different approaches for the phenomenological coupling Hamiltonian can be found: Either one single Hamiltonian with a basis whose dimension equals the sum of photonic modes and excitonic resonances is used. Or a set of independent Hamiltonians, one for each photon mode, is chosen. Both are usually used equivalently for the same kind of multi-photonic systems which cannot be correct. However, identifying the suitable Hamiltonian is difficult when modeling experimental data. By means of numerical transfer matrix calculations, we demonstrate the scope of application of each approach: The first one holds only for the coupling of a single photon state to several excitons, while in the case of multiple photon modes, separate Hamiltonians must be used for each photon mode. (C) 2015 AIP Publishing LLC. [http://dx.doi.org/10.1063/1.4937462]
\end{abstract}

Exciton-polaritons are still an interesting field of research of strong light-matter interaction in solid state systems with bosonic statistics. ${ }^{1-3}$ After various demonstrations of polariton condensation in planar microcavities, ${ }^{4-6}$ microand nanowire systems have raised more interest. ${ }^{7-10}$ In such wire cavities as well as in thick cavities, the photonic system is usually multi-modal where the modes can be of FabryPérot or whispering gallery type. ${ }^{10,11}$ All of those modes can, in principle, couple to excitonic resonances if they are energetically close to the resonance. Furthermore, materials interesting for polaritonics like wide-gap semiconductors as $\mathrm{ZnO}^{12}$ or $\mathrm{GaN}^{13}$ possess several energetically split exciton ground states. Hence, coupling of several cavity photon modes with several excitons has to be considered. ${ }^{14}$

The physics of the coupling between excitons and cavity photons has been described in exact quantum-mechanical models, ${ }^{15,16}$ which consider wave function overlap and quantum degeneracies. However, for evaluations of experimental data, simplified phenomenological models are suitable. Two different approaches of coupled oscillator models are frequently used in the literature to describe the polariton dispersion in systems with $N$ photonic modes and one exciton. However, no distinction is made regarding their appropriability. Either one total $(N+1) \times(N+1)$ system Hamiltonian is used as, e.g., in Refs. 17-20, i.e.,

$$
\mathcal{H}=\left(\begin{array}{ccccc}
E_{X} & V_{1} & V_{2} & \ldots & V_{N} \\
V_{1} & E_{C 1} & 0 & & 0 \\
V_{2} & 0 & E_{C 2} & & 0 \\
\vdots & & & \ddots & \vdots \\
V_{N} & 0 & 0 & \ldots & E_{C N}
\end{array}\right),
$$

with coupling strengths $V_{j} \in \mathbb{R}$. Or, alternatively, the total system Hamiltonian is being composed of a set of $N$ single $2 \times 2$ coupling Hamiltonians for each photonic mode with the exciton separately as used, e.g., in Refs. 7 and 21.
Constructing one system Hamiltonian would give a blockdiagonal $2 N \times 2 N$ matrix with $N$-fold degenerate exciton, i.e.,

$$
\begin{aligned}
\mathcal{H} & =\bigoplus_{j=1}^{N}\left(\begin{array}{cc}
E_{X} & V_{j} \\
V_{j} & E_{C j}
\end{array}\right) \\
& \equiv\left(\begin{array}{ccccccc}
E_{X} & V_{1} & 0 & 0 & \ldots & 0 & 0 \\
V 1 & E_{C 1} & 0 & 0 & \ldots & 0 & 0 \\
0 & 0 & E_{X} & V_{2} & & \vdots & \vdots \\
0 & 0 & V_{2} & E_{C 2} & & & \\
\vdots & & & & \ddots & & \\
0 & 0 & \ldots & & & E_{X} & V_{N} \\
0 & 0 & \ldots & & & V_{N} & E_{C N}
\end{array}\right) .
\end{aligned}
$$

Although approaches 1 and 2 result in different dispersions and deduced coupling strengths, both are used equivalently in the literature for interpretation of experimental data. Even an improper Hamiltonian can provide a sufficient fit. In order to verify which Hamiltonian is the correct approximation, we utilize numerical simulations to study Fabry-Pérot modes in a thick planar microcavity with several photon modes. We stick first to systems with one exciton before we extend the investigation to systems with multiple excitonic resonances.

As long as we restrict the study to the linear response regime, quasi-particle dispersion and electromagnetic wave calculation provide similar descriptions of the modes. ${ }^{22,23}$ In the following, we introduce the wave representation we use and then discuss how to obtain quasi-particle dispersions from it.

We use a complex $4 \times 4$ transfer matrix $\hat{T}$ in order to describe the incoming and outgoing electromagnetic field components $\mathcal{E}$ at both sides $(z$ and $z+d)$ of the structure under consideration. The transfer matrix considers Maxwell's 
equations at all layers and interfaces of a plane parallel structure for a given photon energy $E$ and propagation angle $\theta_{a}$ (angle between surface normal and light propagation in the ambient) or inplane-wavevector $k_{\|}=\frac{E}{\hbar c_{0}} n_{a} \sin \left(\theta_{a}\right)$, respectively ${ }^{24}$

$$
\left(\begin{array}{c}
\mathcal{E}_{s}^{\text {in }}(z) \\
\mathcal{E}_{s}^{\text {out }}(z) \\
\mathcal{E}_{p}^{\text {in }}(z) \\
\mathcal{E}_{p}^{\text {out }}(z)
\end{array}\right)=\hat{T}\left(E, k_{\|}\right)\left(\begin{array}{c}
\mathcal{E}_{s}^{\text {out }}(z+d) \\
\mathcal{E}_{s}^{\text {in }}(z+d) \\
\mathcal{E}_{p}^{\text {out }}(z+d) \\
\mathcal{E}_{p}^{\text {in }}(z+d)
\end{array}\right)
$$

Here, $p$ and $s$ refer to the polarizations parallel and perpendicular to the propagation plane. The constants $c_{0}$ and $\hbar$ are speed of light in the vacuum and reduced Planck constant. Details about the construction of the transfer matrix can be found in the supplementary material. ${ }^{25}$ Optical constants and electronic resonances of the cavity enter $\hat{T}$ through the materials dielectric function. Using the transfer matrix, mode energies can be found by applying the mode condition to each inplane-wavevector $k_{\|}$. For a photonic (polaritonic) mode, it holds (with $\mathcal{E}_{p / s}^{\text {out }} \neq 0$ ) 26,27

$$
\left(\begin{array}{c}
0 \\
\mathcal{E}_{s}^{\text {out }}(z) \\
0 \\
\mathcal{E}_{p}^{\text {out }}(z)
\end{array}\right)=\hat{T}\left(E, k_{\|}\right)\left(\begin{array}{c}
\mathcal{E}_{s}^{\text {out }}(z+d) \\
0 \\
\mathcal{E}_{p}^{\text {out }}(z+d) \\
0
\end{array}\right) \text {. }
$$

Hence, modes are given as (with ker referring to the matrix' kernel)

$$
\begin{gathered}
\left(\begin{array}{l}
\mathcal{E}_{s}^{\text {out }}(z+d) \\
\mathcal{E}_{p}^{\text {out }}(z+d)
\end{array}\right)=\operatorname{ker}\left(\left(\begin{array}{ll}
T_{11} & T_{13} \\
T_{31} & T_{33}
\end{array}\right)\right), \\
\text { ('forward'-traveling waves) } \\
\left(\begin{array}{l}
\mathcal{E}_{s}^{\text {out }}(z) \\
\mathcal{E}_{p}^{\text {out }}(z)
\end{array}\right)=\left(\begin{array}{ll}
T_{21} & T_{23} \\
T_{41} & T_{43}
\end{array}\right)\left(\begin{array}{l}
\mathcal{E}_{s}^{\text {out }}(z+d) \\
\mathcal{E}_{p}^{\text {out }}(z+d)
\end{array}\right) .
\end{gathered}
$$

('backward'-traveling waves)

The sub-transfer matrix considered for the forward-traveling waves becomes singular at the respective mode energy. Hence, we find mode energies by investigating where the determinant of $\left(\begin{array}{ll}T_{11} & T_{13} \\ T_{31} & T_{33}\end{array}\right)$ approaches zero. Generally, such mode energies need to be complex where the imaginary part represents losses causing mode broadening. However, it is sufficient here to neglect the imaginary part.

If no cross-polarization (block off-diagonal elements in the sub-transfer matrix) occurs, as in the isotropic case, the problem can be separated into independent problems for $p$ and $s$-polarization and the mode condition is fulfilled if $T_{11}=0$ ( $s$-polarization) or $T_{33}=0$ ( $p$-polarization), respectively. For finding the mode energies numerically, it is then sufficient to minimize $\left|T_{11}\right|$ and $\left|T_{33}\right|$ with respect to the energy.

Considering a transparent cavity, pure cavity photon modes occur which can be treated as free quasi-particles in 2D. Depending on the optical thickness $n_{\text {cav }} d_{\text {cav }}$ of a cavity layer, one obtains a set of $m \lambda_{C 0} / 2$-cavity modes with mode numbers $m$, where $\lambda_{C 0}=2 n_{\text {cav }} d_{\text {cav }} / m\left(E_{C 0}=m h c_{0} /\right.$ $\left.2 n_{\text {cav }} d_{\text {cav }}\right)$ at $k_{\|}=0$. If the reflectance in the cavity layer against its surroundings is sufficiently high, the broadening of those cavity modes gets small enough (i.e., the cavity photons lifetime becomes large enough) to observe them. The cavity photon energy depends on $k_{\|}$. Considering a 2D quasi-particle dispersion relation, it can be approximated by

$$
E_{C}=\sqrt{E_{C 0}^{2}+\frac{\hbar^{2} c_{0}^{2}}{n_{\mathrm{eff}}^{2}} k_{\|}^{2}} \approx E_{C 0}+\frac{\hbar^{2} k_{\|}^{2}}{2 m_{\mathrm{eff}}} .
$$

The effective refractive index $n_{\text {eff }}$ of the electromagnetic wave description is related to the cavity photon's effective mass $m_{\mathrm{eff}}$ in the parabolic quasi-particle approximation. $E_{C 0}$ is determined by the quantized part of the wavevector $k_{\perp}=$ $k_{z}$ as $E_{C 0}=\hbar c_{0} k_{\perp} / n_{\text {cav }}=m h c_{0} / 2 n_{\text {cav }} d_{\text {cav }}$.

If the cavity material contains electronic (excitonic) resonances in the considered spectral range, polariton modes are observed. With the cavity photon dispersion according to Eq. (5), the polariton energies result directly from applying the coupling Hamiltonian (Eq. (1) or (2), respectively).

As an example system, we consider a symmetric (planar) resonator structure consisting of 29.5 layer pair Bragg mirrors (DBR) surrounding a bulk cavity layer. The structure parameters and optical constants are chosen similar to previous experimental work (cf. Ref. 28) and slightly adapted with respect of the contrast of the refractive indices of the DBR layers and the cavity thickness. The DBR materials are transparent and fulfill the $\lambda / 4$ condition with a central Bragg wavelength of $\lambda \approx 400 \mathrm{~nm}(E=3.1 \mathrm{eV})$. The contrast in the refractive indices $\Delta n=1$ ensures a large energetic width of the Bragg stop band and permits investigation of the cavity photon and polariton modes in a wide spectral range. We use a model-dielectric function for the cavity material which, depending on the considered case, consists of one or two excitonic resonances at $E_{X}=3.35 \mathrm{eV}$ and $3.30 \mathrm{eV}$, respectively, as well as a constant real valued background dielectric function $\varepsilon_{\infty}$ cav . The excitonic resonance is described by a Lorentz oscillator in the dielectric function of the cavity layer

$$
\varepsilon_{\mathrm{cav}}(E)=\varepsilon_{\infty \mathrm{cav}}+\frac{f_{X} E_{X}^{2}}{E_{X}^{2}-E^{2}-i \gamma_{X} E} .
$$

Values used for the broadening $\gamma_{X}$ and oscillator strength $f_{X}$ are listed in Table I. In order to compare the pure photonic system with the coupled one, we set $f_{X}$ either to zero (pure photonic modes) or to the finite value (polariton modes). The simplified approach using Eq. (6) assumes an infinite exciton mass. In the quantization direction, this approximation is justified by the large cavity width. For the inplane direction, the small wavevectors we consider allow neglection of the parabolic dispersion of the 2D free exciton. Practically, this means we assume a degenerate exciton ground state.

We keep the system isotropic throughout the whole consideration here and restrict the further consideration to the $s$ - 
TABLE I. Parameters used for the resonator model.

\begin{tabular}{lcc}
\hline \hline Ambient/substrate refractive index & $n_{a}=n_{s}$ & 1 \\
DBR layer 1 thickness & $d_{\mathrm{DBR} 1}$ & $40 \mathrm{~nm}$ \\
DBR layer 2 thickness & $d_{\mathrm{DBR} 2}$ & $67 \mathrm{~nm}$ \\
DBR layer 1 refractive index & $n_{\mathrm{DBR} 1}$ & 2.5 \\
DBR layer 2 refractive index & $n_{\mathrm{DBR} 2}$ & 1.5 \\
Cavity layer thickness & $d_{\text {cav }}$ & $1.83 \mu \mathrm{m}$ \\
Cavity layer high freq. dielectric const. & $\varepsilon_{\infty \mathrm{cav}}$ & 6.25 \\
Exciton energy & $E_{x}$ & $3.35 \mathrm{eV} ; 3.30 \mathrm{eV}$ \\
Exciton broadening & $\gamma_{X}$ & $1 \mathrm{meV}$ \\
Exciton oscillator strength & $f_{X}$ & $1.5 \times 10^{-3}$ \\
\hline \hline
\end{tabular}

polarization. However, there is no principal difference to the p-polarized case.

In order to study multiple photonic modes, we choose the cavity layer thickness $d_{\text {cav }}$ in the model sufficiently large. Figure 1(a) shows $\left|T_{11}\right|$ to illustrate the dispersion of the $s$ polarized cavity photon modes. Several cavity photon modes can be recognized as minima of $\left|T_{11}\right|$. Note that this illustration does not directly correspond to reflectance spectra ${ }^{22}$ as it represents the modes in terms of well- or ill-definedness of the considered sub-transfer matrix. As shown by the blue solid lines, the dispersion of the photon modes can be well described as 2D quasi-particles using Eq. (5). We only consider the four plotted cavity photon modes with mode numbers $m=\{23,24,25,26\}\left(k_{\perp}\right.$ in the order of $\left.40 \mu \mathrm{m}^{-1}\right)$ in the further discussion. The broadening, i.e. photonic loss, obeys more sophisticated laws, depending on the mode polarization. ${ }^{28}$ However, it is sufficient to neglect it here.

When introducing the (Lorentzian-shaped) exciton as described above, coupling of the photonic modes to the exciton occurs, and the modes evolve as shown in Figs. 1(b) and 1(c). It should be noted that at the energy of the exciton the absolute value of $T_{11}$ gets very large, referring to poles rather than singularities of the considered (sub-)transfer matrix. For the polariton dispersion, we can model the modes by means of either Eq. (1) or Eq. (2) using the photonic mode energies obtained before by applying Eq. (5) and leaving the coupling constants $V_{j}$ as fit parameters. Both approaches are depicted in Figs. 1(b) and 1(c), respectively. It becomes immediately clear that only the ansatz using a series of single Hamiltonians (Eq. (2)) gives the correct description where none of the polariton modes crosses the exciton resonance energy. Thinking in terms of quantized wavefunctions for cavity photon and exciton with cavity resonance at $m \lambda_{C 0} / 2$, it becomes clear that our single exciton resonance used in Eq. (6) is degenerate with respect to $m$ in the quasi-particle description. Coupling with a photonic mode lifts the degeneracy. ${ }^{29}$ Hence, to each mode quantum number $m$, there is one photon mode and one exciton. Consequently, a separate $2 \times 2$ Hamiltonian is needed for each photon mode. The $2 \mathrm{~N}$ dimensional system Hilbert space is indicated by the number of modes visible in $\left|T_{11}\right|$. A comparison to full quantummechanical derivations shows that Eq. (2) is a good approximation. ${ }^{16}$ If the spatial differences of the wavefunctions for the confined excitons and photons are considered, the block off-diagonal elements become finite (but usually small), expressing coupling between modes with different quantum numbers $m$. Remarkably in Fig. 1(b), the spectral density of the modes increases strongly when approaching the exciton resonance energy. This is a consequence of being upper and lower polariton branches to those higher and lower $m$ modes (see supplementary material $^{25}$ ).

It turns out that in the phenomenological approximation Eq. (2) the coupling strengths $V_{j}$ of the exciton to the $j$ th cavity photon are the same for each cavity photon mode. This is expected because the coupling strength is determined by the exciton oscillator strength and the spatial overlap of the mode with the excitons (cavity thickness). ${ }^{30}$

Due to valence band fine structure, several semiconductors have more than one excitonic transition involved in the coupling with cavity photon modes (e.g., $\mathrm{ZnO}$ in Ref. 14). Hence, several excitons have to be considered in the lightmatter coupling Hamiltonian. Exemplarily, we consider the case of $N$ photon modes and two excitons at $3.30 \mathrm{eV}$ and $3.35 \mathrm{eV}$. Similarly to the case with one exciton considered above, the excitons are introduced through the cavity layer's dielectric function. For simplicity and generalization, we still assume isotropic excitons. Figure 2 shows the resulting
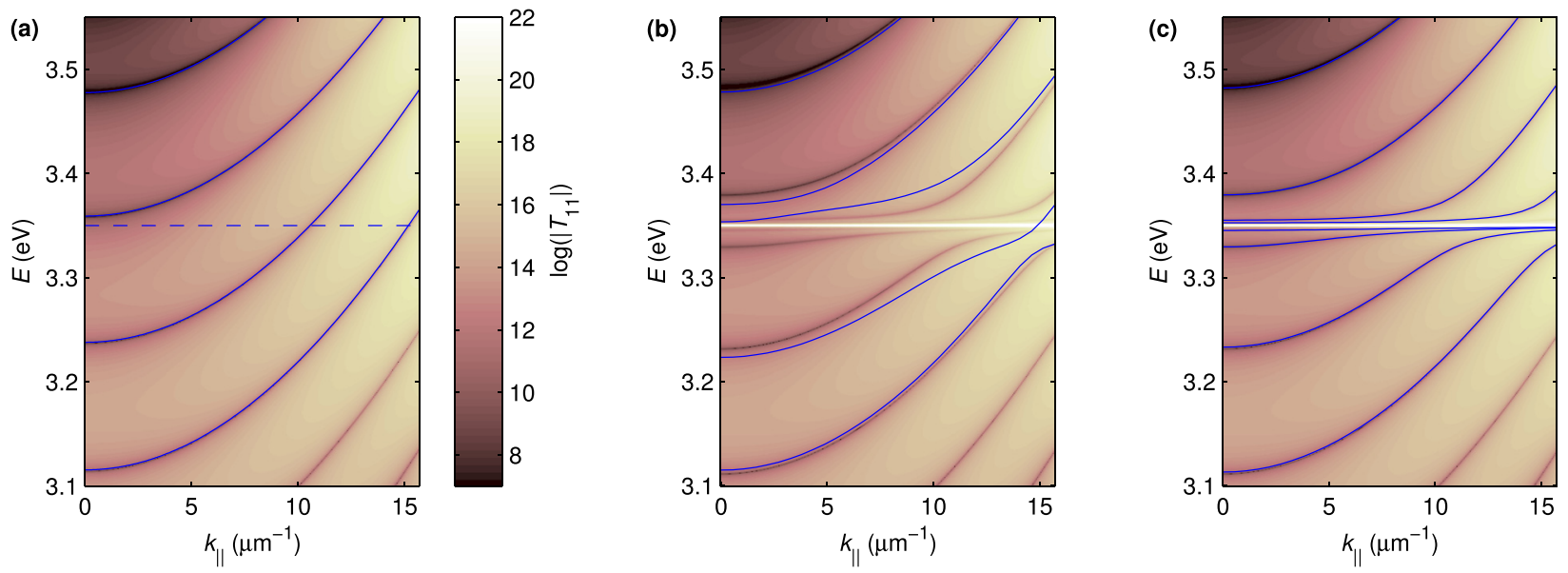

FIG. 1. Logarithmic plot (color bar signifies decimal power) of $\left|T_{11}\right|$ (s-polarization). (a) Pure photonic system with quasi-particle mode dispersions according to Eq. (5) (solid blue lines) and pure exciton energy (dashed blue line, $3.35 \mathrm{eV}$ ). (b) Multi-photonic polariton system with one exciton along with quasi-particle dispersion modeling (blue lines) using Eq. (1) (best fit). (c) The same using Eq. (2). 


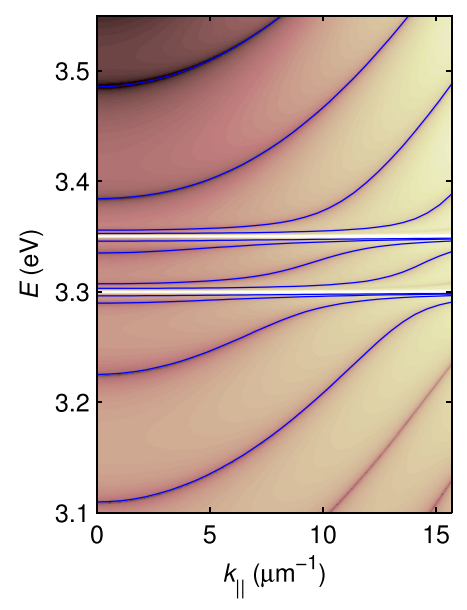

FIG. 2. Logarithmic plot of $\left|T_{11}\right|$ (s-polarization). Multi-photonic polariton system with two excitons at $3.30 \mathrm{eV}$ and $3.35 \mathrm{eV}$ along with quasi-particle dispersion modeling (blue lines) according to Eq. (7). The color scale is the same as in Fig. 1.

dispersion of the polariton modes. As for the single exciton case, no mode crosses any of the excitons. Hence, the excitons must not be treated independently, and only the following Hamiltonian describes the polariton modes correctly, i.e.:

$$
\mathcal{H}=\bigoplus_{j=1}^{N}\left(\begin{array}{ccc}
E_{X 1} & 0 & V_{1 j} \\
0 & E_{X 2} & V_{2 j} \\
V_{1 j} & V_{2 j} & E_{C j}
\end{array}\right) .
$$

This is consistent with Refs. 30 and 31 where only one cavity mode is considered. In general, for each cavity photon, one coupling Hamiltonian involving all excitons has to be applied. Regarding a system of $N$ photon modes and $N_{X}$ excitons, the system must be described by a set of $N$ Hamiltonians of size $\left(N_{X}+1\right) \times\left(N_{X}+1\right)$ (which would result in a single $\left(N N_{X}\right.$ $+N) \times\left(N N_{X}+N\right)$ system Hamiltonian, similar to Eq. (2)). This dimension has also been derived quantum-mechanically. ${ }^{15}$ Also here, the coupling strengths $V_{i j}$ are constant for all $j$, i.e., constant for the coupling of all photonic modes $i$ with a given exciton $j$, but they can differ for different excitonic resonances. $^{3}$

In summary, investigating singularities of electromagnetic transfer matrices for planar microcavities enables rigorous investigations of photonic and polaritonic mode energies and, hence, verification of applied quasi-particle coupling Hamiltonians by considering Maxwell's equations. For a polaritonic system with $N$ photon modes and $N_{X}$ excitons, we showed that $N$ independent $\left(N_{X}+1\right) \times\left(N_{X}+1\right)$ coupling Hamiltonians like Eqs. (2) and (7) must be used, considering each involved cavity photon mode separately. A description with one $\left(N+N_{X}\right) \times\left(N+N_{X}\right)$ system Hamiltonian similar to Eq. (1) is clearly wrong and results in qualitatively incorrect and unphysical polariton dispersion and extracted coupling strengths. As a consequence, $N N_{X}+N$ polariton states occur and the respective $N N_{X}+N$-dimensional Hilbert space consists of $N$ independent sub-spaces, one for each photonic mode. Those findings within simple phenomenological models are consistent with full quantum-mechanical approaches. ${ }^{15}$
The results shown here for Fabry-Perot resonator modes can be transferred directly to the case of whispering gallery modes but with a different photonic mode dispersion. It is generally valid for all photonic resonator modes, especially also the broad edge modes of the Bragg stop band (leaky modes). ${ }^{32}$ Hence, this study clarifies how to correctly describe multi-photonic polariton systems with phenomenological models.

This work was funded by the DFG Grant No. Gru1011/ 20-2. S.R. and T.M. are grateful to the Leipzig graduate school BuildMoNa.

${ }^{1}$ B. Deveaud, Annu. Rev. Condens. Matter Phys. 6, 155 (2015).

${ }^{2}$ V. Kalevich, M. Afanasiev, V. Lukoshkin, D. Solnyshkov, G. Malpuech, K. Kavokin, S. Tsintzos, Z. Hatzopoulos, P. Savvidis, and A. V. Kavokin, Phys. Rev. B 91, 045305 (2015).

${ }^{3}$ C. Ouellet-Plamondon, G. Sallen, F. Jabeen, D. Oberli, and B. Deveaud, Phys. Rev. B 92, 075313 (2015).

${ }^{4}$ J. Kasprzak, M. Richard, S. Kundermann, A. Baas, P. Jeambrun, J. Keeling, F. Marchetti, M. Szymańacuteska, R. André, J. Staehli, V. Savona, P. Littlewood, B. Deveaud, and L. S. Dang, Nature 443, 409 (2006).

${ }^{5}$ T. Guillet, M. Mexis, J. Levrat, G. Rossbach, C. Brimont, T. Bretagnon, B. Gil, R. Butté, N. Grandjean, L. Orosz, F. Réveret, J. Leymarie, J. ZúñigaPérez, M. Leroux, F. Semond, and S. Bouchoule, Appl. Phys. Lett. 99, 161104 (2011).

${ }^{6}$ H. Franke, C. Sturm, R. Schmidt-Grund, G. Wagner, and M. Grundmann, New J. Phys. 14, 013037 (2012).

${ }^{7}$ A. Trichet, L. Sun, G. Pavlovic, N. Gippius, G. Malpuech, W. Xie, Z. Chen, M. Richard, and L. S. Dang, Phys. Rev. B 83, 041302(R) (2011).

${ }^{8}$ Y. Yan and Y. S. Zhao, Adv. Funct. Mater. 22, 1330 (2012).

${ }^{9}$ L. van Vugt, S. Rühle, P. Ravindran, H. Gerritsen, L. Kuipers, and D. Vanmaekelbergh, Phys. Rev. Lett. 97, 147401 (2006).

${ }^{10}$ C. Dietrich, M. Lange, C. Sturm, R. Schmidt-Grund, and M. Grundmann, New J. Phys. 13, 103021 (2011).

${ }^{11}$ T. Nobis, E. Kaidashev, A. Rahm, M. Lorenz, and M. Grundmann, Phys. Rev. Lett. 93, 103903 (2004).

${ }^{12} \mathrm{~F}$. Li, L. Orosz, O. Kamoun, S. Bouchoule, C. Brimont, P. Disseix, T. Guillet, X. Lafosse, M. Leroux, J. Leymarie, M. Mexis, M. Mihailovic, G. Patriarche, F. Réveret, D. Solnyshkov, J. Zúniga-Pérez, and G. Malpuech, Phys. Rev. Lett. 110, 196406 (2013).

${ }^{13}$ P. Bhattacharya, T. Frost, S. Deshpande, M. Z. Baten, A. Hazari, and A. Das, Phys. Rev. Lett. 112, 236802 (2014).

${ }^{14}$ T. Hasegawa, R. Kishimoto, Y. Takagi, T. Kawase, D. Kim, and M. Nakayama, APEX 7, 032003 (2014).

${ }^{15}$ S. Yang and S. John, Phys. Rev. B 75, 235332 (2007).

${ }^{16}$ D. Gerace and L. Andreani, Phys. Rev. B 75, 235325 (2007).

${ }^{17}$ L. Orosz, F. Réveret, S. Bouchoule, J. Zúñiga-Pérez, F. Médard, J. Leymarie, P. Disseix, M. Mihailovic, E. Frayssinet, F. Semond, M. Leroux, M. Mexis, C. Brimont, and T. Guillet, Appl. Phys. Express 4, 072001 (2011).

${ }^{18}$ S. Faure, C. Brimont, T. Guillet, T. Bretagnon, B. Gil, F. Médard, D. Lagarde, P. Disseix, J. Z. J. Leymarie, M. Leroux, E. Frayssinet, J. Moreno, F. Semond, and S. Bouchoule, Appl. Phys. Lett. 95, 121102 (2009).

${ }^{19}$ F. Réveret, P. Disseix, J. Leymarie, A. Vasson, F. Semond, and M. Leroux, Superlattices Microstruct. 52, 541 (2012).

${ }^{20}$ R. Schmidt-Grund, H. Hilmer, A. Hinkel, C. Sturm, B. Rheinländer, V. Gottschalch, M. Lange, J. Zúñiga-Pérez, and M. Grundmann, Phys. Status Solidi B 247, 1351 (2010).

${ }^{21}$ J. Bloch, R. Planel, V. Thierry-Mieg, J. Gérard, D. Barrier, J. Y. Marzin, and E. Costard, Superlattices Microstruct. 22, 371 (1997).

${ }^{22}$ V. Savona, L. Andreani, P. Schwendimann, and A. Quattropani, Solid State Commun. 93, 733 (1995).

${ }^{23}$ D. Solnyshkov, M. Glazov, I. Shelykh, A. Kavokin, E. Ivchenko, and G. Malpuech, Phys. Rev. B 78, 165323 (2008).

${ }^{24} \mathrm{M}$. Schubert, in Handbook of Ellipsometry, edited by H. Tompkins and E. Irene (William Andrew, New York, 2005), Chap. 9, pp. 637-717.

${ }^{25}$ See supplementary material at http://dx.doi.org/10.1063/1.4937462 for construction of the transfer matrices and a more detailed discussion of the number of polariton modes. 
${ }^{26}$ D. Felbacq, Phys. Rev. E 64, 047702 (2001).

${ }^{27}$ D. Bykov and L. Doskolovich, J. Lightwave Technol. 31, 793 (2013).

${ }^{28}$ C. Sturm, H. Hilmer, B. Rheinländer, R. Schmidt-Grund, and M. Grundmann, Phys. Rev. B 83, 205301 (2011).

${ }^{29}$ G. Panzarini and L. Andreani, Phys. Rev. B 60, 16799 (1999).
${ }^{30}$ M. Vladimirova, A. Kavokin, and M. Kaliteevski, Phys. Rev. B 54, 14566 (1996).

${ }^{31}$ F. Réveret, P. Disseix, J. Leymarie, A. Vasson, F. Semond, M. Leroux, and J. Massies, Solid State Commun. 150, 122 (2010).

${ }^{32}$ M. Richard, R. Romestain, R. André, and L. S. Dang, Appl. Phys. Lett. 86, 071916 (2005). 